\title{
Momordica charantia como alternativa terapéutica en la medicina veterinaria
}

\section{Momordica charantia as alternative therapy in veterinary medicine}

Andrade Kujundzic Stefany ${ }^{1}$ Duque Suárez Diego ${ }^{1}$ Jaramillo H. Dumar A. ${ }^{2}$

${ }^{1}$ Medicos Veterinarios Zootecnistas, ${ }^{2}$ MVZ., Esp., cMSc Docente Universidad de los Llanos. Grupo de Investigación en Farmacología Experimental y Medicina Interna - ÉLITE

\author{
dumar.jaramillo@unillanos.edu.co
}

Recibido 17 de Julio 2012, Aceptado 17 Octubre 2012

\section{RESUMEN}

Con el fin de profundizar en el conocimiento de la fitofarmacología colombiana, particularmente aquella presente en la región de los Llanos Orientales, se eligió la especie Momordica charantia para investigar su composición química y así establecer su utilidad en la Medicina Veterinaria y Zootecnia. Para ello se realizó un análisis fitoquímico preliminar, en él se evaluó la presencia de los principales grupos de metabolitos secundarios asociados con actividad biológica, a saber: alcaloides, flavonoides, triterpenos, glucósidos antracénicos, glucósidos cardiogénicos y saponinas. Se detectó la presencia de alcaloides en la Momordica colectada en la Universidad de los Llanos, estos compuestos son ampliamente conocidos por sus propiedades antidiabéticas. Es notable también la presencia de triterpenos, a los cuales se les atribuyen propiedades antiparasitarias, anticancerígenas, antidiabéticas y antialimentarias. Además, se evidencia la presencia de glucósidos cardiogénicos quienes probablemente presentan actividades antivirales. Los resultados de este trabajo son realmente útiles como primera aproximación a la ampliación del conocimiento químico y a la posible utilidad de las especies presentes en esta zona geográfica de Colombia con características medicinales para la salud humana y animal, que permitan una utilización racional de los recursos naturales de nuestro país, como fuente de desarrollo regional y nacional.

Palabras clave: Momordica charantia, fitofarmacología, análisis fitoquímico, metabolitos secundarios. 


\begin{abstract}
In order to further our understanding of phytopharmacology Colombia, particularly those present in the eastern plains region, the species was chosen for further Momordica charantia in chemical composition and thus establish a potential medical utility. This analysis was performed preliminary phytochemical, which evaluated the presence of the main groups of secondary metabolites associated with biological activity, namely: alkaloids, flavonoids, triterpenoids, anthracene glycosides, cardiogenic glycosides and saponins. Was detected the presence of alkaloids in Momordica collected at the University of the Llanos, these compounds are widely known for their antidiabetic properties. It is also remarkable the presence of triterpenes, which are attributed antiparasitic properties, anticancer, anti-HIV, antidiabetic and antifeedant. It also showed the presence of cardiogenic glycosides that may have antiviral activities. The results of this work are really useful as a first approximation to the extension of chemical knowledge and the potential use fullness of the species in this area of Colombia, with medicinal properties to human health and animal-that allow the efficient use of resources country's natural as a source of regional and national development.
\end{abstract}

Keywords: Momordica charantia, phytopharmacology, phytochemical analysis, secondary metabolites.

\title{
INTRODUCCIÓN
}

Las plantas medicinales son usadas como preparados caseros en diferentes comunidades, las cuales han utilizado especies nativas e introducidas desde antes de la época de la colonización. La medicina tradicional es reconocida por la Organización Mundial de la Salud (OMS), desde la incorporación de especies de plantas con un fin terapéutico aplicado a través de las generaciones; es así que la OMS recomienda evaluar la eficacia de estas medicinas naturales con la aplicación de la metodología investigativa desde el punto de vista de ensayos farmacológicos y toxicológicos (Brandão et al., 2008). 
En Colombia debido a la riqueza de su flora son utilizadas las plantas medicinales, por esto; es de gran interés la investigación de ellas, para analizar y estudiar los efectos terapéuticos de las plantas, así como para determinar los compuestos activos responsables de determinada actividad farmacológica, separarlos de las plantas que los contienen, determinar su estructura química, procurar su síntesis, y dar a conocer los resultados de estos estudios.

Actualmente existe un gran interés por el conocimiento y uso de medicinas alternativas, entre las que se destaca la medicina natural. El presente trabajo es un aporte a la construcción de la información relacionada a alternativas terapéuticas con base en plantas y al estudio fitoquímico de Momordica charantia, a la que se le atribuye tradicionalmente propiedades medicinales tanto en medicina humana como en medicina veterinaria. (López, 2006).

Momordica charantia (MC) tiene una larga historia de uso por las comunidades indígenas de la Amazonía. Las hojas de MC se emplean para la diabetes; además como carminativo para el cólico; por vía tópica para el herpes, heridas e infecciones; interna y externamente para parásitos; como emenagogo, y como antiviral para el sarampión, la hepatitis, y estados febriles (Taylor, 2002). En la medicina herbaria brasileña, la Momordica charantia se emplea en el tratamiento de tumores, heridas, reumatismo, malaria, leucorrea, inflamación, problemas menstruales, diabetes, cólicos, fiebre; además para inducir abortos y como afrodisíaco. También se emplea de manera tópica para problemas de la piel, vaginitis, hemorroides, sarna, erupciones pruriginosas, eczema, y lepra (Almeida, 1993). En México, la planta entera se utiliza para la diabetes y la disentería; la raíz es un famoso afrodisíaco (Zamora, 1992). En la medicina herbaria peruana, partes de la hoja se usan para tratar el sarampión, la malaria, y todo tipo de inflamación (Mejia y Rengifo, 1995). En Nicaragua la hoja se utiliza comúnmente para el dolor de estómago, la diabetes, fiebres, resfríos, tos, dolores de cabeza, malaria, afecciones cutáneas, trastornos menstruales, dolores, hipertensión, infecciones, y como coadyuvante en el parto (Coee et al., 1996). Además, MC se ha 
administrado como una medicina popular del Caribe para tratar trastornos leves del tracto gastrointestinal y del sistema urinario (Lans et al., 2006).

La Momordica charantia, conocida como melón amargo; pertenece a la familia de las Cucurbitaceaes y a su vez al género Momordica de la especie charantia, ha sido ampliamente utilizada como planta medicinal (Girón et al., 1991; Lans y Brown, 1998). El nombre latino Momordica significa "morder" (refiriéndose a los bordes dentados de la hoja, que aparecen como si hubieran sido mordidos). Todas las partes de la planta, incluyendo la fruta, presentan un sabor muy amargo. La planta crece en zonas tropicales de Asia, Amazonas, este de África y el Caribe. Se cultiva en todo el mundo para su uso como alimento, así como planta medicinal. MC se ha utilizado tradicionalmente como planta medicinal en los países en desarrollo como Brasil, China, Colombia, Cuba, Ghana, Haití, India, México, Malasia, Nueva Zelanda, Nicaragua, Panamá y el Perú (Satyawati et al., 1987; Yesilada et al., 1999).

MC contiene compuestos químicos que incluyen los glucósidos, saponinas, alcaloides, aceites fijos, triterpenos, esteroides y proteínas (Raman y Lau, 1996). La MC contiene gran cantidad de metabolitos secundarios activos, principalmente de tipo alcaloide y triterpenoide como lo muestra la Tabla $N^{\circ} 1$. Se han descrito varios metabolitos secundarios como momorcharinas, momordenol, momordicilinas, momordicinas, momordicininas, momordin, momordolol, charantinas, charines, criptoxantina, cucurbitinas, cucurbitacinas, cucurbitanes, cicloartenoles, diosgenina, ácidos elaeostearicos, eritrodiol, ácido galacturónico, ácido gentísico, goyaglicosidos, goyasaponinas, multiflorenol (Husain et al., 1994; Xie et al., 1998; Yuan et al., 1999; Parkash et al., 2002). Estos compuestos se presentan en todas las partes de la planta (Murakami et al., 2001).

El análisis nutricional de $\mathrm{MC}$ indica que este vegetal es rico en fibra, calcio, potasio, hierro y vitaminas A y C (USDA, 2008). Además, la pulpa alrededor de las semillas de la fruta madura es una buena fuente de licopeno carotenoide (Yen y Hwang, 1985). El componente amargo de la MC se ha caracterizado por tener cuatro glucósidos cucurbitaneos, momordicosidos $\mathrm{K}$ y L, y momordicinas I y II 
(Okabe, et al., 1982; Yasuda, et al., 1984) además contiene fitoquímicos, como el polipéptido-p (Khanna, et al., 1981) y el charantin (Lotlikar, et al., 1966).

Tabla 1. Principales alcaloides y triterpenos de la especie Momordica charantia

\begin{tabular}{|c|c|c|c|}
\hline $\begin{array}{l}\text { GRUPO DE } \\
\text { METABOLITO }\end{array}$ & TIPO QUIMICO & $\begin{array}{l}\text { PARTE DE LA } \\
\text { PLANTA } \\
\end{array}$ & PLANTA ORIGEN \\
\hline \multirow{3}{*}{ Alcaloides } & Charina & $\begin{array}{c}\text { Frutos } \\
\text { (inmaduros) } \\
\end{array}$ & Egipto \\
\hline & $\begin{array}{c}\text { Zeatina } \\
\text { Zeatina riboside }\end{array}$ & Semillas & India \\
\hline & Momordicina & $\begin{array}{l}\text { Hojas } \\
\text { Frutos }\end{array}$ & Brasil \\
\hline \multirow{30}{*}{ Triterpenos } & Amyrin, $\beta$ : & \multirow{6}{*}{$\begin{array}{l}\text { Aceite de } \\
\text { semilla }\end{array}$} & \multirow{6}{*}{ Japón } \\
\hline & $\begin{array}{c}\text { Cucurbita-5-24-dien-3- } \\
\text { beta-ol,10-alpha: }\end{array}$ & & \\
\hline & Cycloartenol & & \\
\hline & $\begin{array}{l}\text { Cycloartenol,24- } \\
\text { methylene: }\end{array}$ & & \\
\hline & Multiflorenol & & \\
\hline & Taraxerol & & \\
\hline & $\begin{array}{l}\text { Cucurbita-5-24-dien-19- } \\
\text { al,2-3-dihydroxy: }\end{array}$ & Planta entera & Bangladesh \\
\hline & $\begin{array}{c}\text { Cucurbita-5-24-diene,3- } \\
\text { beta-7-beta- } 23- \\
\text { trihydroxy: } 7 \text {-o-beta-d- } \\
\text { glucoside }\end{array}$ & \multirow{4}{*}{ Hojas } & \multirow{4}{*}{ Nigeria } \\
\hline & $\begin{array}{c}\text { Momordica cucurbitane } \\
3 \\
\end{array}$ & & \\
\hline & $\begin{array}{c}\text { Momordica cucurbitane } \\
6 \\
\end{array}$ & & \\
\hline & Momordicin 8 & & \\
\hline & Cucurbitacin B & \multirow[t]{2}{*}{ Semillas } & \multirow[t]{2}{*}{ No se indica } \\
\hline & Cucurbitacin K & & \\
\hline & $\begin{array}{c}\text { Cycloart-cis-23-ene-3- } \\
\text { beta-25-diol }\end{array}$ & \multirow{7}{*}{ Semillas } & \multirow{7}{*}{ Japón } \\
\hline & Erythrodiol & & \\
\hline & Karounidiol & & \\
\hline & $\begin{array}{l}\text { Karounidiol,dihydro: } 7- \\
\text { oxo: }\end{array}$ & & \\
\hline & Karounidiol,iso: & & \\
\hline & $\begin{array}{l}\text { Karounidiol, iso: 3-para- } \\
\text { methoxy-benzoate }\end{array}$ & & \\
\hline & Karounidiol-3-benzoate & & \\
\hline & Goyaglycoside A & \multirow{10}{*}{ Fruto } & \multirow{10}{*}{ Japón } \\
\hline & Goyaglycoside B & & \\
\hline & Goyaglycoside C & & \\
\hline & Goyaglycoside D & & \\
\hline & Goyaglycoside E & & \\
\hline & Goyaglycoside F & & \\
\hline & Goyaglycoside G & & \\
\hline & Goyaglycoside H & & \\
\hline & Goyasaponin I & & \\
\hline & Goyasaponin ii & & \\
\hline
\end{tabular}




\begin{tabular}{|c|c|c|c|}
\hline $\begin{array}{c}\text { GRUPO DE } \\
\text { METABOLITO }\end{array}$ & TIPO QUIMICO & $\begin{array}{l}\text { PARTE DE LA } \\
\text { PLANTA }\end{array}$ & PLANTA ORIGEN \\
\hline & Goyaglycoside D & \multirow{8}{*}{ Fruto } & \multirow{8}{*}{ Japón } \\
\hline & Goyaglycoside E & & \\
\hline & Goyaglycoside F & & \\
\hline & Goyaglycoside G & & \\
\hline & Goyaglycoside $\mathrm{H}$ & & \\
\hline & Goyasaponin I & & \\
\hline & Goyasaponin ii & & \\
\hline & Goyasaponin ii & & \\
\hline & Gypsogenin & \multirow{2}{*}{ No se indica } & \multirow{2}{*}{ Egipto } \\
\hline & Oleanolic acid & & \\
\hline & Momorcharaside A & \multirow{2}{*}{ Semillas } & \multirow{2}{*}{ China } \\
\hline & Momorcharaside B & & \\
\hline & $\begin{array}{l}\text { Momordica charantia } \\
\text { triterpene glycoside }\end{array}$ & Cotiledones & India \\
\hline & Momordicinin & \multirow{4}{*}{ Fruto } & \multirow{4}{*}{ Pakistan } \\
\hline & Momordicilin & & \\
\hline & Momordicin & & \\
\hline & Momordol & & \\
\hline & Momordicin I & \multirow[b]{2}{*}{ Hojas } & \multirow[b]{2}{*}{$\begin{array}{l}\text { India } \\
\text { Nigeria }\end{array}$} \\
\hline & Momordicin ii & & \\
\hline \multirow[t]{22}{*}{ Triterpenos } & Momordicine 1 & Frutos (inmaduros) & No se indica \\
\hline & Momordicine I & \multirow{3}{*}{ Hojas } & \multirow{3}{*}{$\begin{array}{l}\text { Japón } \\
\text { India }\end{array}$} \\
\hline & Momordicine ii & & \\
\hline & Momordicine iii & & \\
\hline & Momordicoside A & $\begin{array}{l}\text { Semillas } \\
\text { Frutos }\end{array}$ & $\begin{array}{l}\text { China } \\
\text { Japón }\end{array}$ \\
\hline & Momordicoside B & Semillas & \multirow{17}{*}{ Japón } \\
\hline & Momordicoside C & $\begin{array}{c}\text { Semillas } \\
\text { Frutos } \\
\end{array}$ & \\
\hline & Momordicoside D & \multirow{15}{*}{ Frutos (inmaduros) } & \\
\hline & Momordicoside $\mathrm{E}$ & & \\
\hline & Momordicoside E' & & \\
\hline & Momordicoside E-1 & & \\
\hline & Momordicoside Ex & & \\
\hline & Momordicoside F & & \\
\hline & Momordicoside F & & \\
\hline & Momordicoside F-1 & & \\
\hline & Momordicoside F-2 & & \\
\hline & Momordicoside G & & \\
\hline & Momordicoside $\mathrm{H}$ & & \\
\hline & Momordicoside I & & \\
\hline & Momordicoside J & & \\
\hline & Momordicoside $\mathrm{K}$ & & \\
\hline & Momordicoside L & & \\
\hline
\end{tabular}

Fuente: Modificado de (Taylor, 2002)

En el fruto y las semillas de $M C$ se han identificado compuestos como el Momordin, ácido oleanólico-3-O-monodesmosido y ácido oleanólico-3-O- 
glucurónido (Murakami, et al., 2001). Muchos cucurbitaneos tipo triterpenoides han sido aislados de frutos (Okabe, et al., 1982; Murakami, et al., 2001; Harinantenaina, et al., 2006), semillas (Miyahara, et al., 1981; Okabe, et al., 1980), tallos (Chang, et al., 2006; Cheng, et al., 2008), raíces (Chen, et al., 2008), hojas y ramas (Yasuda, et al., 1984; Chang, et al., 2006).

\section{MATERIALES Y MÉTODOS}

El estudio se realizó en la Universidad de los Llanos ubicada en Colombia, departamento del Meta. En la ciudad de Villavicencio, en la vereda Barcelona (Vía Puerto López Km. 7). Con latitud N 404'25.81” y latitud O 73³5'09.73", altitud de 465 metros sobre el nivel del mar, temperatura promedio de $270 \mathrm{C}$, precipitación anual entre 1900 y $2300 \mathrm{~mm}$ y humedad relativa de $80 \%$.

Para obtener el material vegetal se recolectaron $10 \mathrm{~kg}$ de hojas, frutos, semillas, tallos y raíces de Momordica charantia, en la Granja Barcelona de la Universidad de los Llanos. La preparacion del extracto de Momordica charantia, se realizó siguiendo la metodología propuesta por Sanabria (1983). La obtención del extracto etanólico (Figura 1) se realizó así: El material vegetal recolectado se cortó y se secó en una estufa marca Siemens de aire recirculante a $40^{\circ} \mathrm{C}$ durante $72 \mathrm{~h}$. (Figura 2); luego el material seco fue molido hasta pulverización, como se muestra en la Figura 3. Estos procedimientos fueron realizados en el Laboratorio de Nutrición animal de la Universidad de los Llanos. Luego se tomó el material molido para la técnica de percolación continúa hasta agotamiento, con etanol al 98\% (Figura 4); el líquido percolado se filtró con papel filtro de 20-25 $\mu \mathrm{m}$ y concentro a $40^{\circ} \mathrm{C}$ en un evaporador rotatorio marca Buchi R-124, llevándose a deshidratación finalmente en baño de María. Este procedimiento fue realizado en el Laboratorio de química de la Universidad de los Llanos. 


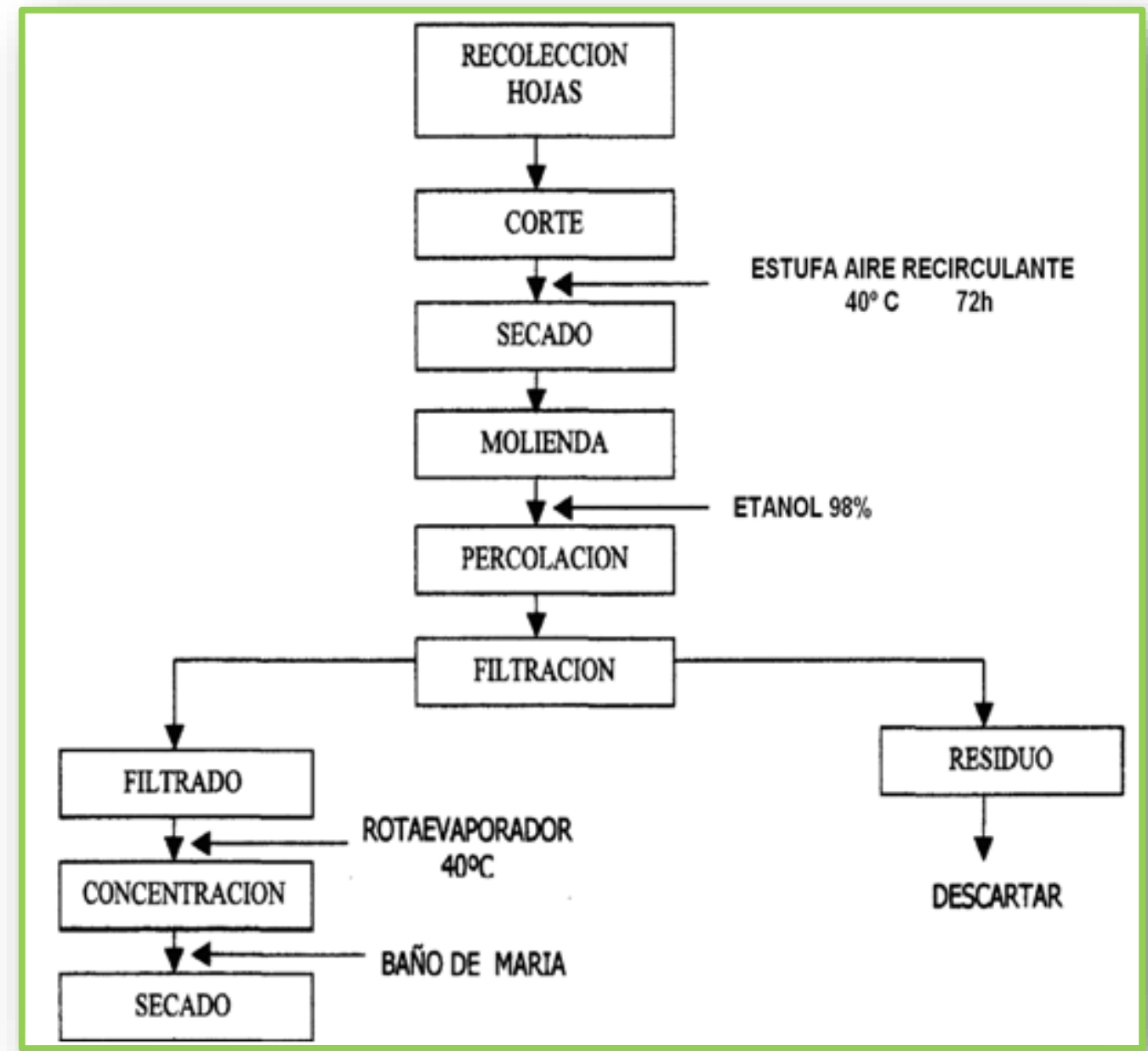

Figura 1. Obtención del extracto etanólico (Tomado de: Sanabria, 1983).

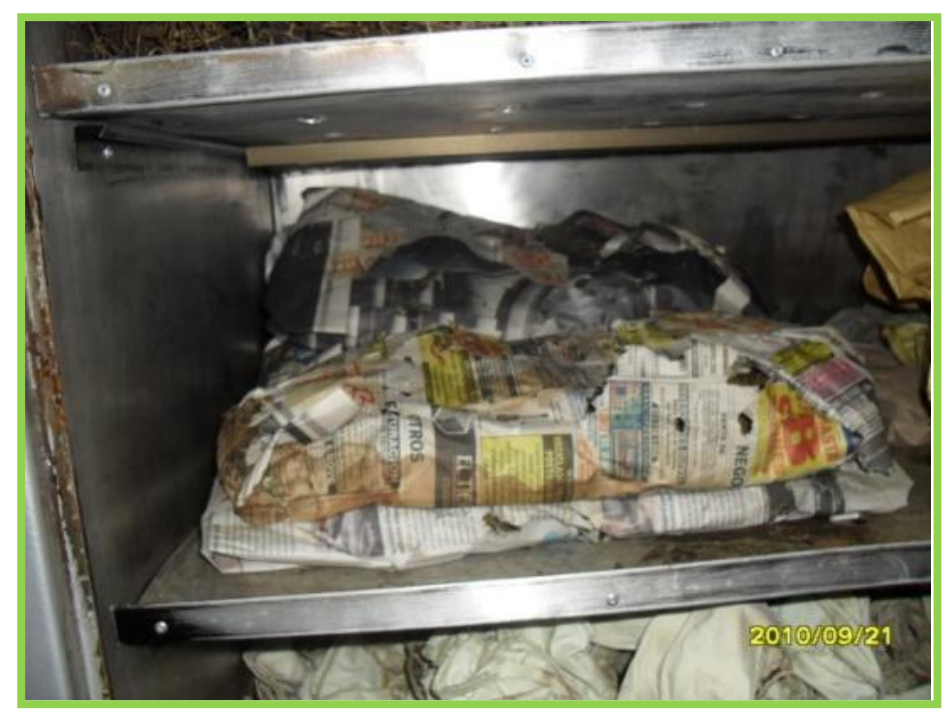

Figura 2. Secado. 


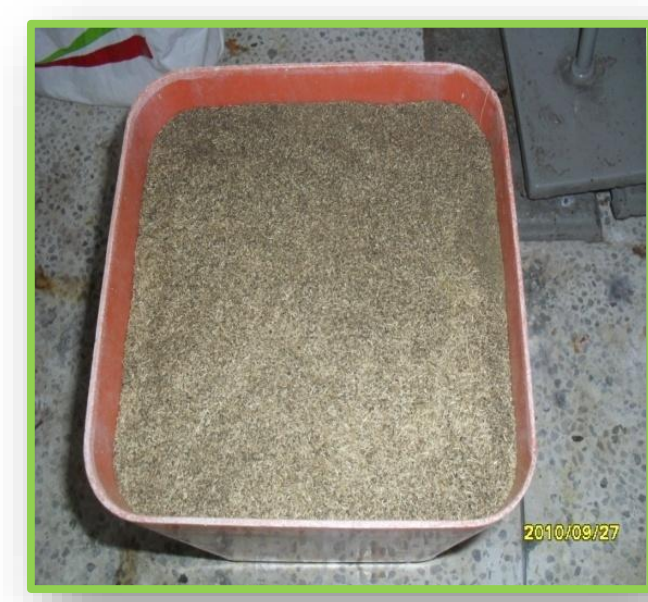

Figura 3. Material molido.

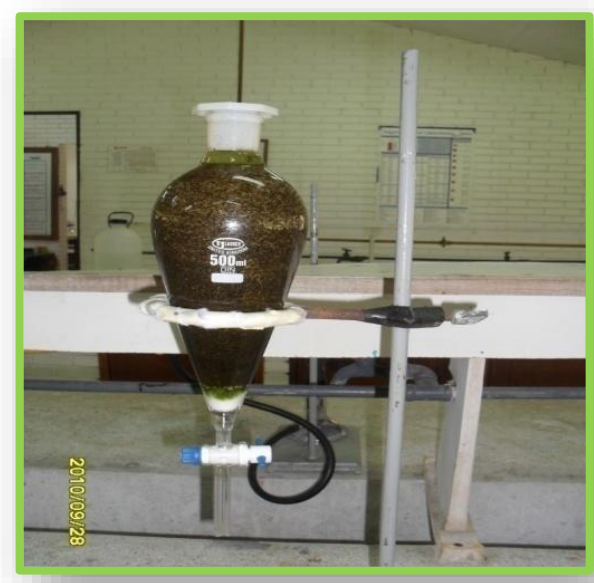

Figura 4. Percolación con etanol al $98 \%$

El análisis fitoquímico preliminar del material vegetal de Momordica charantia, se realizó siguiendo la metodología propuesta por Sanabria (1983). El líquido percolado en etanol al $98 \%$ se filtró y se evaporo el solvente del residuo, el producto obtenido se dividió en tres residuos; con el residuo I se midieron alcaloides y con el residuo II esteroides y/o triterpenoides, flavonoides, naftoquinonas y/o antraquinonas, saponinas y taninos. El extracto III se concentró a volumen medio y este se utilizó para detectar lactonas terpenicas, cumarinas y cardiotónicos, como se muestra en la Figura 5. Este procedimiento fue realizado en el Laboratorio de química de la Universidad de los llanos.

La marcha fitoquímica e identificación de los metabolitos secundarios de Momordica charantia, se realizó siguiendo la metodología propuesta por Ahmed et al., (2005). Para la realización de la marcha fitoquímica (identificación de los metabolitos secundarios) se utilizó la técnica de cromatografía en capa delgada y la precipitación de soluciones. Para ambas técnicas se tomó 1 gramo del extracto etanólico de MC y se disolvió en $10 \mathrm{ml}$ de etanol al 98 \% (Solución 1) en un vaso de precipitado de $50 \mathrm{ml}$ como se muestra en la Figura 6.

En una cromatoplaca o cromatofolio de $5 \mathrm{~cm}$ de largo $\times 1 \mathrm{~cm}$ de ancho se colocó una gota de la solución del extracto etanólico con una pipeta Pasteur de vidrio moldeada a capilar como se muestra en la Figura 7. Después de realizar el montaje de las cromatoplacas se procedió a llevarlas a la cámara de fases (Figura 
8). Se utilizó como fase estacionaria silica gel y como fase móvil un eluyente de cloroformo metanol 90:10.

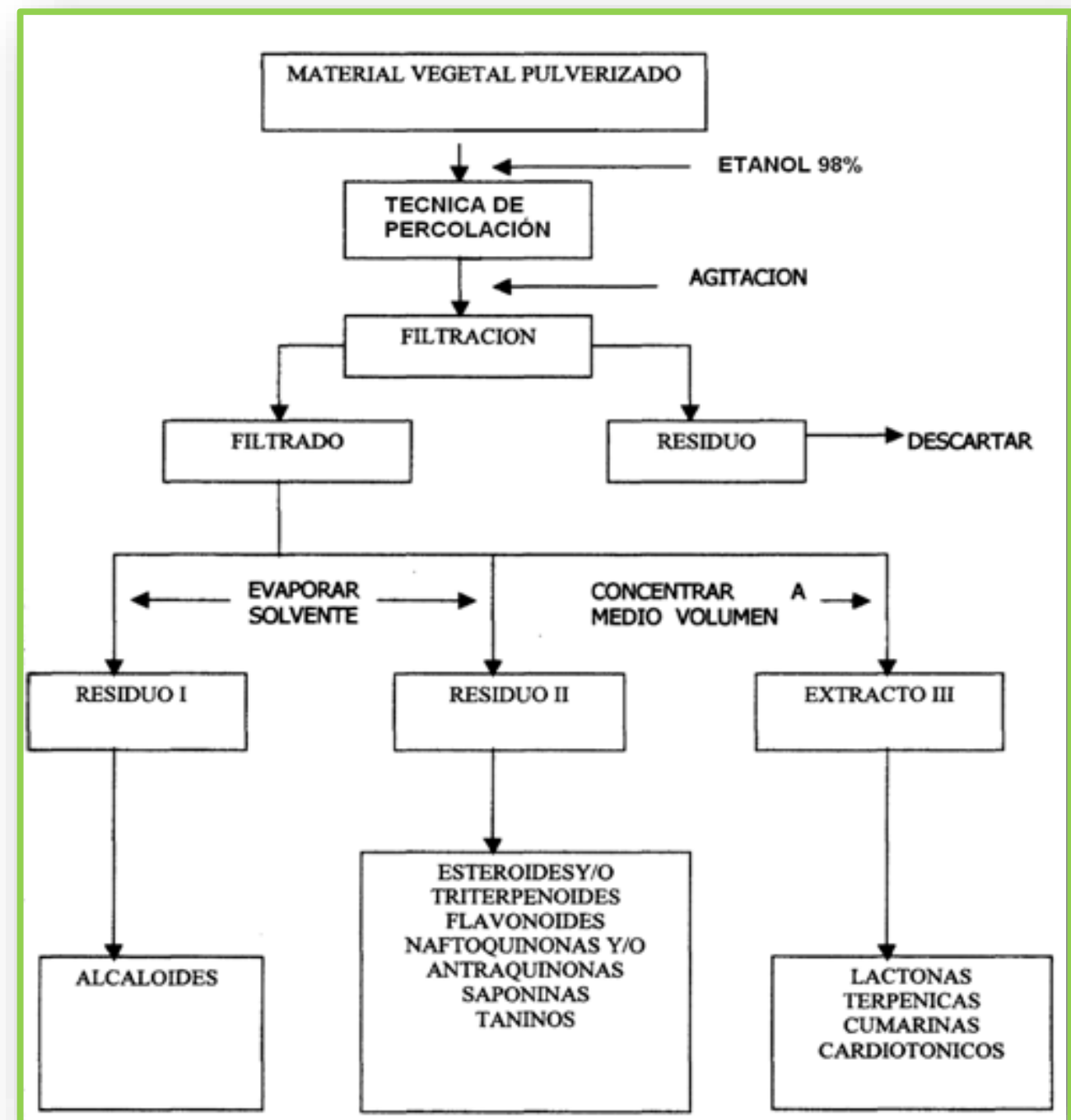

Figura 5. Análisis fitoquímico preliminar de las hojas de Momordica charantia.

(Tomado de Sanabria, 1983)

Para la determinación de alcaloides se utilizó como patrón la quinidina. Luego de colocar la cromatoplaca en la cámara se esperó que arrastrara eluyendo por polaridad los diluyentes del extracto casi hasta el final, se marcaron las clorofilas flotantes. La placa se retiró y se reveló con los reactivos correspondientes de Permanganato de potasio y Reactivo de Dragendorff. Luego la placa se calentó un poco en el horno hasta que seco para luego ser llevado a la luz ultravioleta. El 
resultado fue fluorescencia a los rayos UV, coloración amarrilla. Esta coloración determina la presencia de alcaloides en el extracto etanólico de MC.

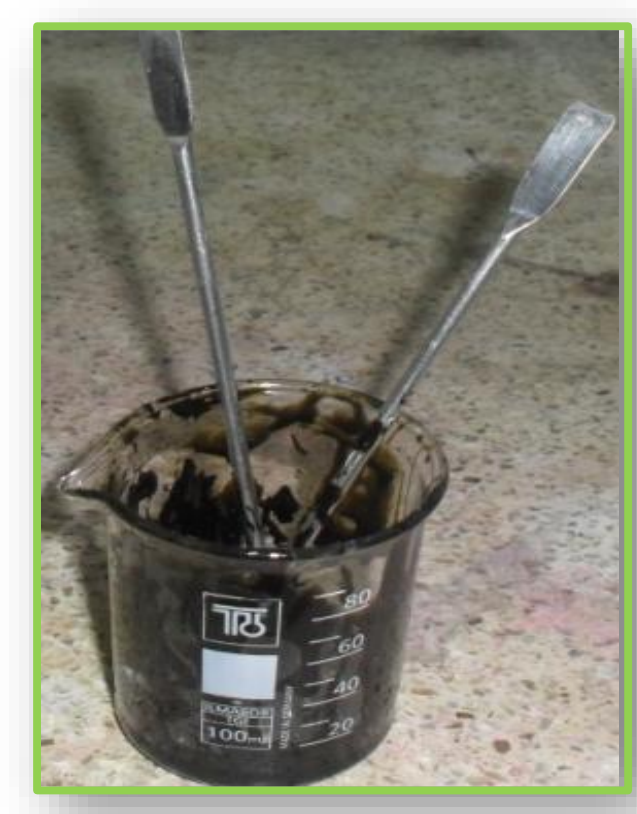

Figura 6. Extracto etanólico de Momordica charantia

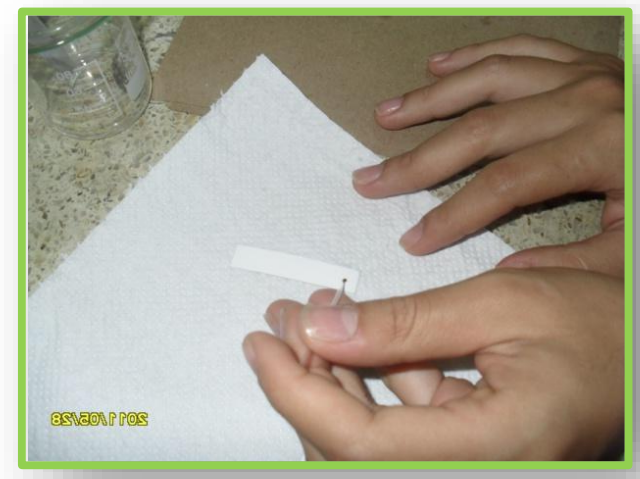

Figura 7. Montaje de las cromatoplacas.

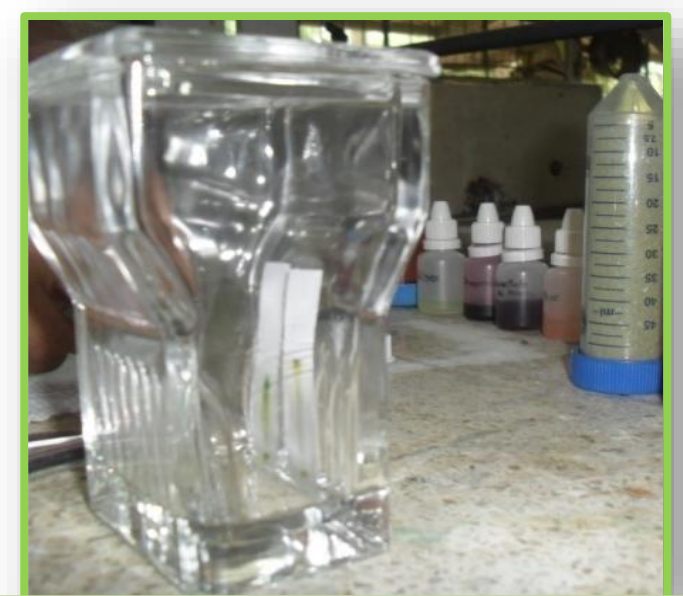

Figura 8. Cromatoplacas en la cámara de fases

Para la determinación de flavonoides se utilizó como patrón la flavona y como revelador el reactivo NP. Se utilizaron dos soluciones, la solución A con Reactivo de productos naturales (1\% en metanol) y la solución B con solución de PEG 4000 
al $5 \%$ en etanol. Se asperjo con la solución $A$, se dejó secar y luego se asperjo con la solución $B$, se dejó secar y se observó a la luz ultravioleta. No se observaron zonas fluorescentes determinando la ausencia de flavonoides en el extracto.

Para la determinación de triterpenos se utilizó el Reactivo de Godin y dos soluciones, la solución $A$ con Vainillina y la solución $B$ con ácido perclórico al $3 \%$ en etanol. Se asperjo la cromatoplaca con la solución A seguida de la solución B. Se calentó a $100^{\circ} \mathrm{C}$ y se observó a la luz UV. Como resultado se observó un poco de fluorescencia lo cual indica un resultado positivo para triterpenos.

Para la determinación de glucósidos cardiogénicos se utilizó como patrón la digitalina. Se trabajaron dos cromatoplacas. El primer recorrido se realizó con éter de petróleo - acetato de etilo y el segundo recorrido con cloroformo-metanol. Como revelador se utilizó el reactivo de Kedde o de Raymond. Se prepararon dos soluciones. La Solución 1 se disolvió $0,1 \mathrm{gr}$ de ácido 3,5-dinitrobenzoico en $10 \mathrm{ml}$ de metanol y la solución $2 \mathrm{NaOH}$ al $10 \%$ en etanol. No se presentó ninguna reacción o coloración lo cual indica la ausencia de glucósidos cardiogénicos en el extracto.

Para la determinación de glucósidos antracénicos se utilizó como patrón la cascara sagrada. Se sumergió una cromatoplaca con el extracto en una mezcla de benceno-acetato de etilo-ácido acético (75:24:1). Se dejo secar y se observó a la luz UV. Se reveló la cromatoplaca con una solución de $\mathrm{KOH}$ al $10 \%$ en etanol. No presentó ninguna coloración de fluorescencia, por lo tanto, es negativo para glucósidos antracénicos.

Para realizar los ensayos de precipitación, fue necesario preparar diferentes diluciones. Para esto se tomó la solución 1 y se prepararon las soluciones 2, 3 y 4 así: SIn 2, 1:9 extracto-etanol, SIn 3, 1:9 extracto-etanol, SIn 4, 4:40 extractoetanol (Figura 9). Para cada tubo se trabajaron $5 \mathrm{ml}$ de la SIn 4. 


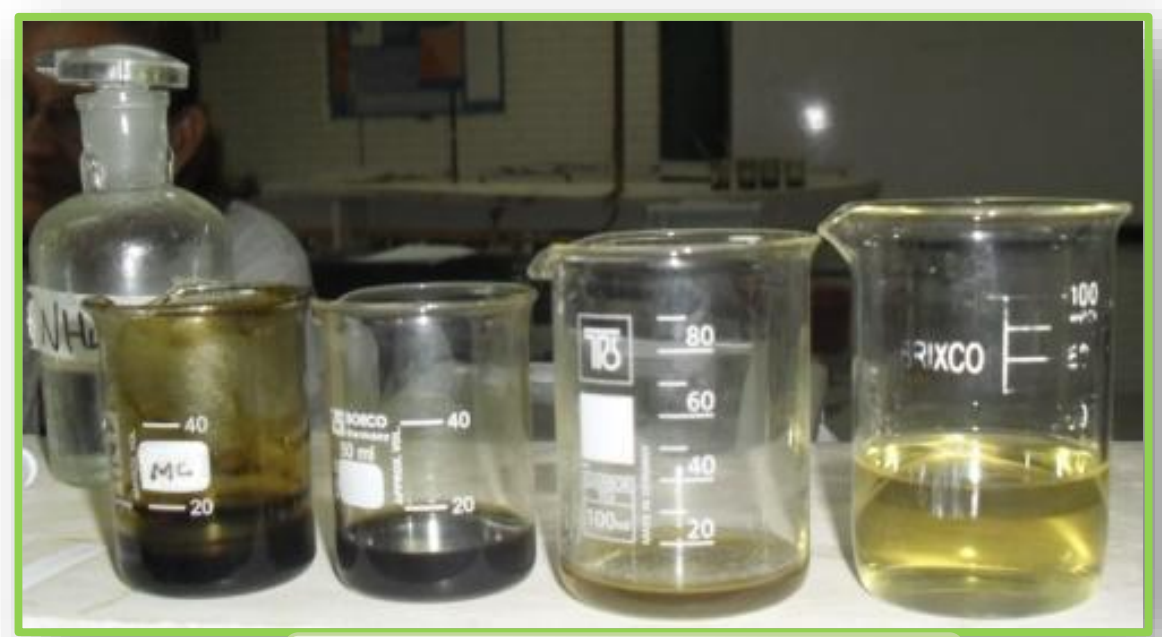

Figura 9. Soluciones 1, 2, 3 y 4

\section{RESULTADOS Y DISCUSIÓN}

Para la determinación de alcaloides se utilizó como patrón la quinidina (Figura 10). Los alcaloides fueron precipitados en forma amorfa con los siguientes reactivos:

- Tubo 1: Reactivo de Mayer: Produce un precipitado de color crema en la presencia de alcaloides como se observa en la figura 10, para el extracto de MC no se observa el precipitado color crema; por lo tanto, se determina ausencia de alcaloides mediante la reacción de Mayer (Figura 11).

- Tubo 2: Reineckato de amonio (alcaloides con amonio cuaternario): En presencia de alcaloides produce un precipitado de color crema o blanco. No se observa precipitado en el extracto de MC, negativo para alcaloides con amonio cuaternario.

- Tubo 3: Reactivo de Dragenforff: Ante la presencia de alcaloides produce un precipitado de color rojo anaranjado. Nótese la formación del precipitado rojo anaranjado en el extracto de MC confirmando la presencia de alcaloides mediante el reactivo de Dragenforff.

- Tubo 4: Reactivo de Valser: En presencia de alcaloides se genera un precipitado de color crema. No se observa este precipitado en el extracto de MC; por lo tanto se determina la ausencia de alcaloides por el reactivo de valser. 


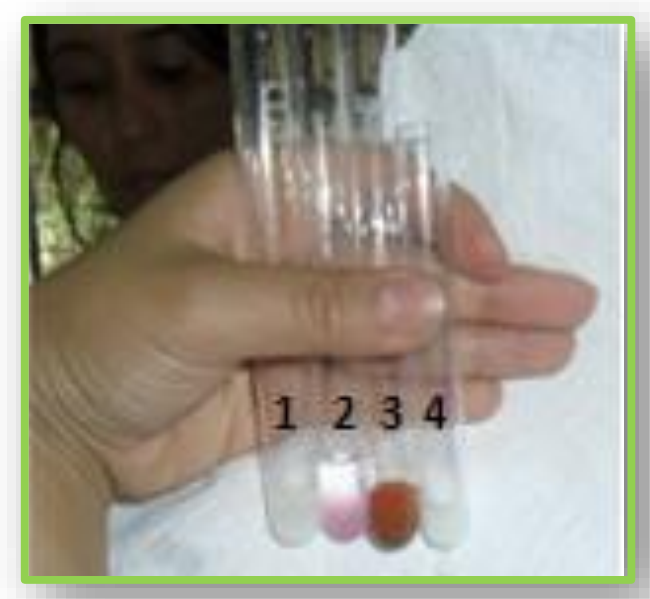

Figura 10. Patrón de quinidina.

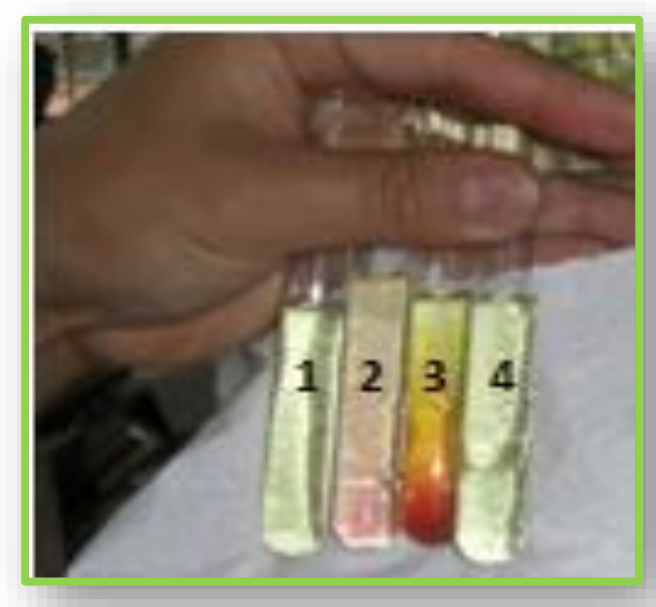

Figura 11. Prueba de precipitación para alcaloides en MC

Para la determinación de flavonoides se utilizó como patrón la flavona y la Reacción de Shinoda o de Willstater (ensayo de magnesio-ácido clorhídrico). Se adiciono a la solución del extracto un trozo pequeño de $\mathrm{Mg}$ y tres gotas de ácido clorhídrico concentrado. Normalmente si es positivo debe mostrar una coloración rojo-violeta concentrado. Resultado reacción de Shinoda: se observa una coloración amarillo-rosado, pero no se obtiene un precipitado rojo violeta concentrado como se observa en la Figura 12 (Tubo P); por lo tanto, esta prueba se considera negativa para flavonoides.

Para la determinación de Glucósidos antracénicos se utilizó como patrón la cascara sagrada. Como reactivo se utilizó una solución de $5 \%$ hidróxido de sodio y $2 \%$ amoniaco. Se tomaron $5 \mathrm{ml}$ del extracto y se le adiciono el reactivo. Normalmente si es positivo debe mostrar una coloración rojiza. Como resultado se obtiene una coloración verde clara. (Figura 13). Determinando la prueba como negativa para glucósidos antracénicos en el extracto de MC. 


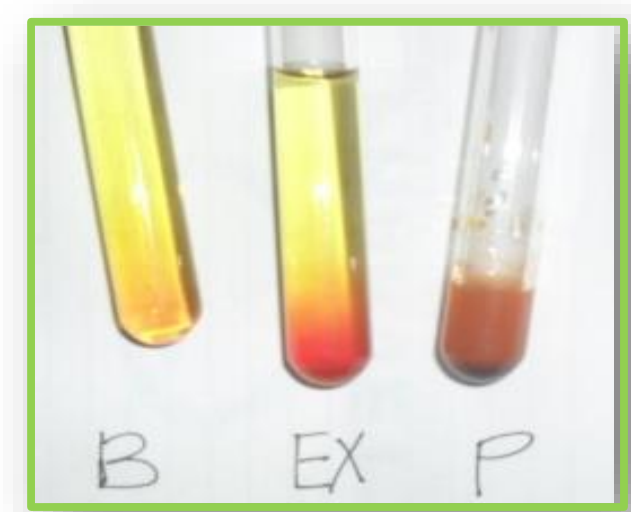

Figura 12. Prueba de precipitación para flavonoides en MC.

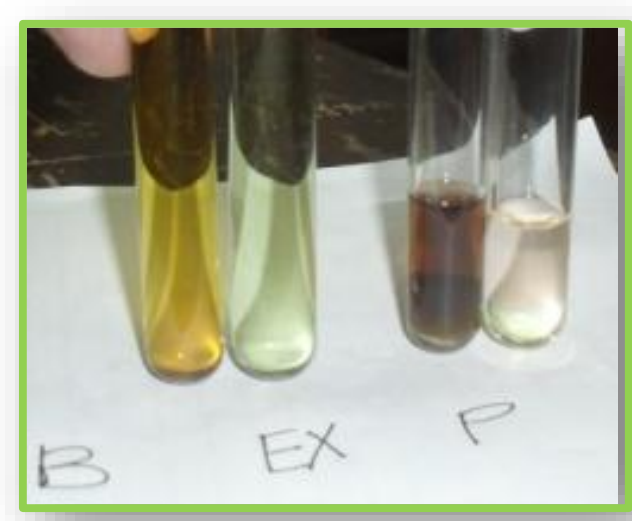

Figura 13. Prueba de precipitación para glucósidos antracénicos en MC.

Para la determinación de glucósidos cardiogénicos se utilizó como patrón la digitalina y se realizaron las siguientes reacciones:

- Tubo 1: Reacción de Kedde o de Raymond: Produce un precipitado de color rojo violeta en la presencia de lactona. Para el extracto de MC se observó precipitado color rojo violeta (Figura 14) por lo tanto se determina positivo a compuestos con lactona en su estructura.

- Tubo 2: Reacción de sellér - Kiliani: Produce un precipitado de color verde intenso en la presencia de desoxiazúcares. Se obtiene una coloración rojiza para el extracto de $\mathrm{MC}$, se considera negativo para glucósidos cardiogénicos en presencia de desoxiazúcares (Figura 15).

Para la prueba de precipitación de saponinas se utilizaron como patrones positivos las siguientes diluciones. Para la primera solución se tomó un extracto positivo a saponinas, agua y se agito hasta producir espuma. En la segunda solución se trabajó una dilución del extracto y sangre en proporción 1:1, se agito observándose Hemolisis. Para la solución del extracto de MC y agua al agitar no se observó formación de espuma. Para la solución del extracto de MC y sangre, no se observó hemolisis por lo tanto se considera negativo para la presencia de saponinas (Figura 16). 


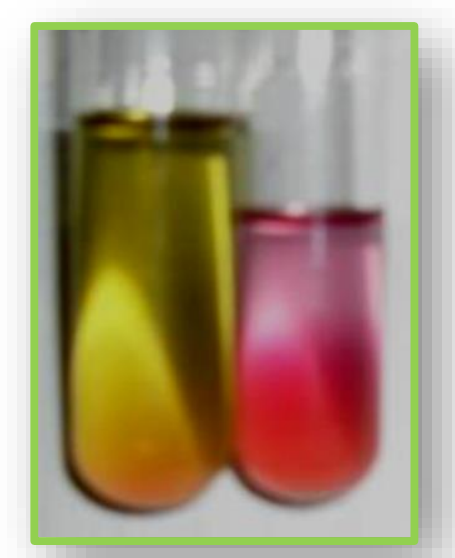

Figura 14. Reacción de Kedde 0 de Raymon en MC

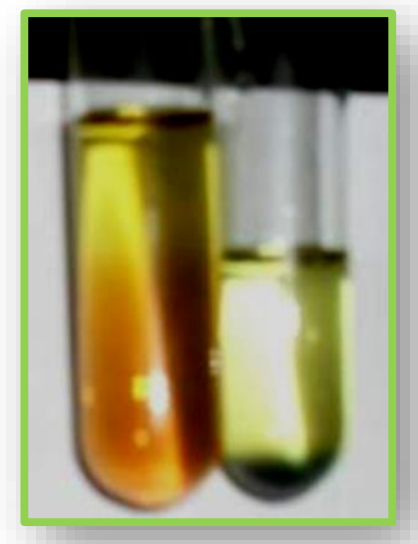

Figura 15. Reacción de sellér - Kiliani) en MC.

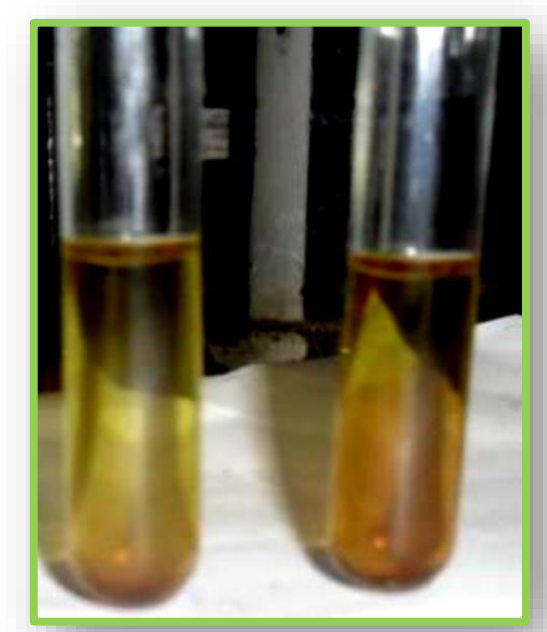

Figura 16. Prueba de precipitación para saponinas.

El estudio fitoquímico de la especie Momordica charantia colectada en la Universidad de los Llanos, mostro la presencia de metabolitos secundarios de tipo alcaloide por medio de las pruebas de Cromatografía en capa delgada y precipitación, como se muestra en la Tabla 2. También se hallaron metabolitos de tipo triterpenoide en la prueba de precipitación. Se hallaron metabolitos secundarios de la planta MC recolectada en la universidad de los llanos semejantes a los descritos en la literatura. 
Tabla 2. Estudio fitoquímico de la especie Momordica charantia

\begin{tabular}{|c|c|c|c|}
\hline $\begin{array}{l}\text { METABOLITO } \\
\text { SECUNDARIO }\end{array}$ & PATRON BASE & $C C D^{*}$ & PRECIPITACION \\
\hline ALCALOIDES & Quinidina & + & $\begin{array}{c}\text { +(Alcaloides sin } \\
\text { amonio cuaternario) }\end{array}$ \\
\hline FLAVONOIDES & Flavona & - & - \\
\hline TRITERPENOS & --------- & + & --------- \\
\hline $\begin{array}{c}\text { GLUCOSIDOS } \\
\text { ANTRACENICOS }\end{array}$ & Cascara sagrada & - & - \\
\hline $\begin{array}{c}\text { GLUCOSIDOS } \\
\text { CARDIOGENICOS }\end{array}$ & Digitalina & - & $\begin{array}{c}\text { + (Compuestos con } \\
\text { lactona en su } \\
\text { estructura })\end{array}$ \\
\hline SAPONINAS & 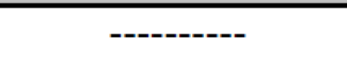 & --------- & - \\
\hline
\end{tabular}

Los resultados de la marcha fitoquímica de la $\mathrm{MC}$ colectada en la granja Barcelona de la Universidad de los Llanos mostro la presencia de alcaloides, triterpenos y glucósidos cardiogénicos. En cuanto a los glucósidos cardiogénicos es necesario realizar pruebas más específicas, como las de tipo espectrométrico, para confirmar realmente su presencia, ya que no se halló una lectura realmente significativa para ratificar la presencia de ellos en la planta, pero tampoco para negar su ausencia total, que puede estar relacionada con una presencia baja. Posiblemente la MC de la universidad de los llanos comparte propiedades fitofarmacológicas con respecto a las reportadas en la literatura. De esta forma se corrobora la presencia de estos metabolitos como se describe en la Tabla 2.

En cuanto al mecanismo de acción de cada uno de los metabolitos encontrados en la planta, no hay claridad en la literatura sobre que método utilizan estos para hacer su efecto; por consiguiente es necesario realizar estudios posteriores, donde se pueda definir caracterizaciones más específicas para la búsqueda y purificación de los metabolitos secundarios, que permitan llegar a la tipificación detallada de 
cada tipo de alcaloide, triterpeno y glucósido que contenga la MC de la universidad de los llanos. Así mismo, realizar pruebas más específicas como las espectrofotométricas (ultravioleta-visible, infrarrojo, resonancia magnética nuclear de protón y de carbono-13), la cromatografía de intercambio iónico HPLC y la electroforesis, para aislar cada componente químico de los grupos de metabolitos que conlleven a determinar su presencia.

La MC ha sido utilizada como medicina alternativa por mucho tiempo, pero realmente para trabajarla en la terapéutica medica veterinaria se debe tener en cuenta la sobrevivencia medica clínica y el diagnostico medico en el momento de definir sus efectos para determinada enfermedad. Es preciso conocer la interacción dentro del organismo animal de todos y cada uno de los compuestos de la MC, se sugiere la implementación de modelos biológicos que corroboren su actividad terapéutica en animales, estos modelos deben ser realizados siguiendo las normas de bioética.

Es necesario conocer de manera específica cómo interactúan los componentes de la MC, para poder utilizarlo como medicamento alternativo en la terapéutica medica veterinaria como tratamiento de enfermedades de los diferentes sistemas orgánicos como las citadas en la revisión bibliográfica; como hipoglicemiante en la diabetes mellitus tipo I y II, antineoplásico, antihipertensivo, antimicrobial, etc. Al manejar una medicina basada en evidencias no se puede trabajar con supuestos sino con comprobados, se necesita seguir estudiando estos compuestos para saber si es un metabolito el que genera los efectos terapéuticos o son varios, determinar si se puede hacer purificaciones o se tiene que manejar la planta como tal.

\section{CONCLUSIONES}

Al realizar la revisión técnica - científica de la literatura nacional e internacional sobre MC, se determinó la potencialidad de su uso como fitofármaco en la terapéutica medica veterinaria, fortaleciendo las producciones orgánicas, 
principalmente mitigando procesos como resistencias y residualidades de medicamentos alopáticos en el tratamiento de enfermedades animales.

La cromatografía en capa delgada y las pruebas colorimétricas, permitieron comprobar la presencia cualitativa de los siguientes grupos de metabolitos secundarios en MC: alcaloides, triterpenos y glucósidos cardiogénicos. Es necesario corroborar la presencia de estos realizando caracterizaciones más específicas para la búsqueda y purificación de los metabolitos secundarios de la MC, aislándolos de los grupos de metabolitos para determinar el mecanismo de acción específico de cada uno de ellos.

Las investigaciones etnofarmacológicas son un paso fundamental para la búsqueda de nuevas sustancias bioactivas. Los estudios fitofarmacológicos posteriores deben incluir la forma de uso tradicional y la posibilidad de sinergismo de los distintos compuestos para una validación científica coherente.

Este estudio es pionero en la región, es el primero que se realiza sobre la $\mathrm{MC}$ en el llano, por lo tanto es relevante e importante seguir haciendo la caracterización de la planta, hasta llegar a la descripción especifica del mecanismo de acción de cada uno de sus metabolitos; debido a la determinación de estos en la planta y su posible uso como estrategia terapéutica en la medicina veterinaria y por tanto sea una alternativa eficiente su utilización, aprovechando que es una planta nativa en la región.

Se puede decir que en un futuro la medicina homotoxicológica puede llegar a tener la importancia según el soporte científico que se le dé a la misma.

\section{REFERENCIAS BIBLIOGRÁFICAS}

1. Ahmed M. S., Calle, J. Reportes manual de laboratorio de farmacognosia y fitoquímica. Departamento de farmacia de la Universidad Nacional de Colombia. Bogotá. 2005.

2. Almeida. DE. Plantas medicinais brazileiras, Conhecimentos populares e científicos. Hemus Editora Ltda. Sao Paulo. 1993.

3. Brandão, M.G., Zanetti, N., Oliveira, P., Grael, C.F., Santos, A.P., Monte-Mór L.M. Brazilian medicinal plants described by $19^{\text {th }}$ century European naturalists 
and in the Official Pharmacopoeia. Journal of Ethnopharmacology, 120: 14148. 2008.

4. Chang, C. I., Chen, C. R., Liao, Y. W., Cheng, H. L., Chen, Y. C., Chou, C. H. Cucurbitane-type triterpenoids from Momordica charantia. Journal of Natural Products, 69: 1168-1171. 2006.

5. Cheng, H. L., Huang, H. K., Chang, C. I., Tsai, C. P., Chou, C. H. A cell-based screening identifies compounds from the stem of Momordica charantia that overcome insulin resistance and activate AMP-activated protein kinase. Journal of Agricultural and Food Chemistry, 56: 6835-6843. 2008.

6. Chen, J., Tian, R., Qiu, M., Lu, L., Zheng, Y., Zhang, Z. Trinorcucurbitane and cucurbitane triterpenoids from the roots of Momordica charantia. Phytochemistry, 69, 1043-1048. 2008.

7. Coee, FG., Anderson, GJ. Ethnobotany of the Garifuna of Eastern Nicaragua. Econ Bot, 50 (1): 71-107. 1996. Sch Pharm Univ Connecticut Storrs Ct 06268 USA.

8. Giron, L.M., Freire, V., Alonzo, A., Caceres, A., Ethnobotanical survey of the medicinal flora used by the Caribs of Guatemala. Journal of Ethnopharmacology, 34: 173-187. 1991.

9. Harinantenaina, L., Tanaka, M., Takaoka, S., Oda, M., Mogami, O., Uchida, M., Asakawa, Y., Momordica charantia constituents and antidiabetic screening of the isolated major compounds. Chemical and Pharmaceutical Bulletin, 54: 1017-1021. 2006.

10. Husain, J., Tickle, I.J., Wood, S.P., Crystal structure of momordin, a type I ribosome inactivating protein from the seeds of Momordica charantia. FEBS Letters, 342: 154-158. 1994.

11. Khanna, P., Jain, S. C., Panagariya, A., Dixit, V. P. Hypoglycemic activity of polypeptide-p from a plant source. Journal of Natural Products, 44: 648-655. 1981.

12. Lans, C., Brown, G. Observations on ethnoveterinary medicines in Trinidad and Tobago. Preventive Veterinary Medicine, 35: 125-142. 1998.

13.Lans, C., Turner, N., Brauer, G., Lourenco, G., Georges, K. Ethnoveterinary medicines used for horses in Trinidad and in British Columbia, Canada. Journal of Ethnobiology and Ethnomedicine, 2: 31. 2006.

14. López LT. Plantas medicinales con actividad hipoglicemiante. Características, administración y efectos adversos. 25 (5). 2006.

15. Lotlikar, M. M., Rajarama Rao, M. R. Pharmacology of a hypoglycaemic principle isolated from fruit of Momordica charantia Linn. Industrial Journal of Pharmacology, 28: 129-133. 1966.

16. Mejia, K., Rengifo, E. Plantas medicinales de uso popular en la amazonia peruana. Aeci And Ilap. Lima. 1995.

17. Miyahara, Y., Okabe, H., Yamauchi, T. Studies on the constituents of Momordica charantia L. II. Isolation and characterization of minor seed glycosides, Momordicosides C, D, and E. Chemical and Pharmaceutical Bulletin (Tokyo) 29: 1561-1566. 1981.

18. Murakami, T., Emoto, A., Matsuda, H., Yoshikawa, M., Medicinal foodstuffs. Part XXI. Structures of new cucurbitane-type triterpene glycosides, goyaglycosides-a, -b, -c, - $d$, - $-e,-f,-g$, and $-h$, and new oleanane-type triterpene 
saponins, goyasaponins I, II, and III, from the fresh fruit of Japanese Momordica charantia L. Chemical \& Pharmaceutical Bulletin (Tokyo), 49: 5463. 2001.

19. Okabe, H., Miyahara, Y., Yamauchi, T., Miyahara, K., Kawasaki, T. Studies on the constituents of Momordica charantia L. I. Isolation and characterization of momordicosides A and B, glycosides of a pentahydroxy-cucurbitane triterpene. Chemical and Pharmaceutical Bulletin (Tokyo), 28: 2753-2762. 1980.

20. Okabe, H., Miyahara, Y., Yamauchi, T. Studies on the constituents of Momordica charantia L. IV. Characterization of the new cucurbitacin glycosides of the immature fruits. (2). Structures of the bitter glycosides, momordicosides K and L. Chemical and Pharmaceutical Bulletin (Tokyo), 30: 4334-4340. 1982.

21.Parkash, A., Ng, T.B., Tso, W.W., Purification and characterization of charantin, a napin-like ribosome-inactivating peptide from bitter gourd (Momordica charantia) seeds. Journal of Peptide Research, 59: 197-202. 2002.

22. Raman, A., Lau, C. Anti-diabetic properties and phytochemistry of Momordica charantia L. (Cucurbitaceae). Phytomedicine, 2: 349-362. 1996.

23. Sanabria A. Análisis fitoquímico preliminar. Universidad Nacional de Colombia, Facultad de Ciencias, Departamento de Farmacia, Bogotá,1-61. 1983.

24. Satyawati, G.V., Gupta, A.K., Tandon, N., Medicinal plants of India. Indian Council of Medical Research, New Delhi, India, 262 p. 1987.

25. Taylor, L. Technical data report for bitter melon (Momordica charantia). En: Herbal Secrets of the Rainforest. $2^{\text {nd }}$ edition Sage Press. 2002.

26. USDA. National Nutrient Database for Standard Reference, Release 21. 2008. Disponible en: http://www.nal.usda.gov/fnic/foodcomp/cgi-bin/list nut edit.pl/

27. Xie, H., Huang, S., Deng, H., Wu, Z., Ji, A., Study on chemical components of Momordica charantia. Zhong Yao Cai. 21: 458-459. 1998.

28. Yasuda, M., Iwamoto, M., Okabe, H., Yamauchi, T. Structures of momordicines I, II, and III, the bitter principles in the leaves and vines of Momordica charantia L. Chemical and Pharmaceutical Bulletin (Tokyo), 32: 2044-2047. 1984.

29. Yen, G. C., Hwang, L. S. Lycopene from the seeds of ripe bitter melon (Momordica charantia) as a potential red food colorant. II. Storage stability, preparation of powdered lycopene and food applications. Journal of Chinese Agricultural Chemical Society, 23: 151-161. 1985.

30. Yesilada, E., Gurbuz, I., Shibata, H. Screening of Turkish antiulcerogenic folk remedies for anti-Helicobacter pylori activity. Journal of Ethnopharmacology 66, 289-293. 1999.

31. Yuan, Y.R., He, Y.N., Xiong, J.P., Xia, Z.X. Three-dimensional structure of beta-momorcharin at $2.55 \mathrm{~A}$ resolution. Acta Crystallographica Section DBiological Crystallography, 55: 1144-1151. 1999.

32. Zamora M. Medicinal plants used in some rural populations of Oaxaca, Puebla and Veracruz, Mexico. J Ethnopharmacol, 35 (3): 229-257. 1992. Cent Inv Forest Agrop Dis Fed Mexico 04110 México. 